\title{
UNIVERSIDAD, CIENCIA Y TECNOLOGIA EN ARGENTINA
}

\author{
MARIO ALBORNOZ *
}

\begin{abstract}
Al retornar la democracia, la Universidad pública argentina tuvo que enfrentar varios desafíos simultáneos y aún pendientes, como emergentes específicos de la crisis sufrida por la sociedad, en su conjunto, durante la dictadura militar y los largos años de inestabilidad política. Dos de ellos revisten un carácter esencial: la recuperación de los valores propios de la cultura universitaria y la definición de su perfil institucional frente a las características actuales de la sociedad. En ambos desafios la ciencia desempeña un papel central.
\end{abstract}

\section{La ciencia en la cultura universitaria}

La cultura universitaria argentina, heredera de la Reforma y de diversas tradiciones de pensamiento, implica el ejercicio de la autonomía, el cogobierno y la libertad de cátedra, entre otros valores esenciales, pero comprende también algunos dilemas no bien resueltos a lo largo de los años, tal como la opción entre los modelos de la Universidad napoleónica y de la Universidad científica, inspirada en von Humboldt.

La Universidad argentina recibió de la Reforma una fuente impronta que se constituyó en un paradigma para muchos países de América Latina. En 1918, poco después de las primeras elecciones bajo la ley que instauraba el sufragio universal, secreto y obligatorio, los estudiantes de la Universidad de Córdoba reaccionaron ante los cambios en el ambiente y la necesidad de la propia institución de adaptarse a las nuevas reglas de juego sociales, políticas y económicas.

"El movimiento de Córdoba resulta...del desajuste entre las caducas estructuras y valores de wha Universidad heredera de la colonia y las expectativas de un estudiantado que se percibe a si mismo como la vanguardia de una modernidad libertaria, ilustrada, urbana, científica y racionalista".(BRUNNER, 1991)

Con un sentido trascendente, los protagonistas de aquel proceso consideraban a la Universidad como un ámbito desde el que sería posible acelerar y extender las

* Universidad Nacional de Buenos Aires. 
transformaciones a toda la sociedad. La Reforma de la Universidad contenía una poderosa energía de renovación social que tuvo fuerza suficiente como para expandirse desde la provincia de Córdoba hacia toda la Argentina, primero, y hacia América Latina después. En aquella década fueron Perú, Chile, Cuba, Colombia, Guatemala y Uruguay los países que recogieron con mayor entusiasmo el legado reformista. En la del ' 30 se agregarían Brasil, Paraguay, Bolivia, Ecuador, Venezuela y México.(PORTANTIERO, 1978)

Aquella vanguardia ilustrada, científica, urbana y racionalista, al decir de Brunner, no resolvió de modo unívoco el problema del papel de la ciencia en el modelo de Universidad que de ella surgió, ya que ciertos elementos incluidos en su sistema de valores colisionaban con los que sustentaba la también naciente "comunidad científica".

Por otra parte, la Reforma no es la única tradición de la que se nutre la Universidad argentina, ya que está también inspirada en el modelo napoleónico, el cual, si bien surgió de la concepción liberal de la Revolución Francesa, fue expresión de una voluntad política que se tradujo en que el Estado tomara a su cargo, en forma absoluta, la responsabilidad de la educación superior, como así también la regulación de las profesiones.

La Universidad, en consecuencia, se vioestrechamente involucrada con lascorporaciones profesionales bajo una concepción jerarquizada de la sociedad, según la cual la obtención de un título se constituía en un elemento casi indispensable para el éxito en la vida social y laboral. La Universidad "profesionalista" tiene, así, profundas raíces que la vinculan con demandas sociales, si bien en el contexto de un determinado modelo económico y su correspondiente estructuración societaria.

También la tradición de la Universidad científica inspirada en Humboldt tiene algún grado de supervivencia en la Universidad argentina, en cuyos claustros nació y se desarrolló una importante comunidad científica que fue capaz de brindar al país y a toda América Latina los únicos Premios Nobel en Ciencias con que cuenta hasta el presente.

Estas tradiciones dejan un saldo a ser tenido en cuenta para la nueva reforma que la Universidad argentina trata hoy de abordar. La Reforma deja el legado de una energía transformadora que procura adaptar la institución universitaria a las demandas renovadoras de la sociedad, en un cuadro de valores todavía vigente. El modelo napoleónico ayuda a recordar la importancia del Estado como elemento de sustentación de la educación superior, cuyo papel como actor central debe ser reconocido. Del modelo de Humboldt es recuperable la concepción de la Universidad científica, en un marco de autonomía y de libertad académica.

El modelo humboldtiano, implícito en el discurso de Bernardo Houssay y de otros investigadores que constituyeron una verdadera "aristocracia" de la ciencia en Latinoamérica, adquiere hoy nueva vigencia por las posibilidades que ofrece para rediseñar las Universidades en función de las demandas de la sociedad, sin que esto signifique necesariamente la opción por una "Universidad para pocos". 


\section{Valor crítico del conocimiento}

Las circunstancias actuales, caracterizadas por la revolución del paradigma tecnológico dominante, el agotamiento de un modelo productivo, la revalorización de las funciones innovadoras y el surgimiento de nuevas condiciones competitivas en escenarios internacionales ampliados, con poderosas transformaciones en todos los planos de la actividad social, ponen en evidencia el valor crítico del conocimiento científico y tecnológico.

Esto se traduce, para las Universidades, en la necesidad de dar respuesta a nuevas demandas, tanto en la formación de profesionales dotados de habilidades específicas, como en la producción de conocimiento científico básico y orientado hacia la tecnología, así como en el establecimiento de fuertes vínculos operativos con otros actores sociales.

El hecho de que las Universidades deban desempeñar una función destacada en la sociedad como centros dedicados a la investigación científica y a la producción de conocimiento tecnológico transferible al sector productivo, significa en el momento presente que, además del valor "esencial" asignado a la ciencia en el modelo inspirado en von Humboldt, ella desempeña un papel fundamental en la definición del lugar que a las Universidades corresponde ocupar en lo que actualmente se denomina como "sistema nacional de innovación" o "sistema ciencia-tecnología-industria". Esto exige cambios en la conducta de los actores e impone un sistema de valores diferentes a los tradicionales en las comunidades científica y académica, fenómeno que sin lugar a dudas ya se está produciendo.

Frente al desafío que hoy representa el desarrollo tecnológico para la sociedad argentina, sería suicida desaprovechar el potencial de $\mathrm{I}+\mathrm{D}$ con que cuentan las Universidades, el cual representa más de la mitad del número de los investigadores activos en el país. Por el contrario, se requiere la articulación de políticas que permitan estimular y aprovechar, con una perspectiva de largo plazo, la capacidad científica universitaria, tanto por la demanda de conocimiento tecnológico, como por la necesaria e íntima vinculación entre la investigación científica y la calidad de la enseñanza.

\section{Ciencia y tecnología en la Universidad}

Las Universidades públicas, en su conjunto, son las instituciones de mayor importancia desde el punto de vista del desarrollo científico y tecnológico y del estímulo a las capacidades innovadoras del país. Ellas constituyen la más grandereserva de investigadores, ya que reúnen -según el censo de 1988 - el $54,7 \%$ del personal científico y tecnológico (cuadro 1). Una sola Universidad -la de Buenos Aires- posee el 14,7\% del PCT nacional y casi triplica el número de los investigadores de la institución que le sigue: la CONEA.

La productividad de los investigadores universitarios, medida a través de un indicador clásico, como es el de las publicaciones en revistas científicas internacionales con arbitraje, es superior al de la media de otras instituciones de $\mathrm{I}+\mathrm{D}$. 
En efecto, de acuerdocon los resultados de una investigación realizada por la Secretaría de Ciencia y Técnica de la UBA sobre el "Citation Index, 1991", por cada 100 investigadores en el país, 13 publican al menos un artículo anual de tales características. Tal rendimiento se duplica para los investigadores de la UBA y la UNLP.

El cuadro 2 permite observar el fuerte aumento de las publicaciones científicas de las Universidades de mayor producción, así como la declinación de los institutos del CONICET vinculados a la UBA. En forma simétrica, el importante incremento de la UNLP expresa, además, la incorporación estadística de los centros del CONICET platenses a dicha Universidad. Entre 1980 y 1989 la UBA aumentó su participación porcentual dentro del conjunto de las Universidades, pasando del $20,23 \%$ al $22,29 \%$ del total de las publicaciones. La UNLP, con la salvedad hecha, aumentó más fuertemente su participación, pasando del $5,43 \%$ al $9,77 \%$.

El cuadro 3 muestra la producción de tres de las más importantes Universidades latinoamericanas. La Universidad Central de Venezuela tiene una producción más baja y estabilizada; la Universidad Nacional Autónoma de México tiene un desempeño similar al de la UBA, aunque resulta inferior en relación a su tamaño. La Universidad de San Pablo, en cambio, la aventaja, si bien se debe tener presente que su presupuesto, como se verá con más detalle, aproximadamente sextuplica al de la UBA.

\section{Universidades y política científica nacional}

Desde hace años existe cierto grado de "conflicto institucional" respecto al modo de inclusión de las Universiđades en las políticas nacionales de ciencia y tecnología. Esto se deriva, en parte, de la represión hacia las Universidades ejercida por los gobiernos militares, que tuvieron -entre otras-como consecuencia la creación y fortalecimiento de centros de investigación extra-universitarios. La "onda expansiva" de surgimiento de institutos del CONICET entre 1976 y 1983 es una prueba de ello.

En otra medida, sin embargo, el conflicto es estructural y estaba implícito ya en la misma creación del CONICET, cuyas funciones no siempre han estimulado el desarrollo de la investigación en la Universidad, sino que han tendido a separarla de ella, contribuyendo así -paradójicamente- al refuerzo de los aspectos más crudamente "profesionalistas".

Como consecuencia de esta tensión, los investigadores universitarios, pese a ser los que demuestran mayor productividad, son también los que disponen de peor financiamiento, ya que en forma directa apenas se asigna a las Universidades e $18,1 \%$ del presupuesto nacional para ciencia y tecnología. A la Universidad de Buenos Aires que, como se ha dicho, tiene casi el $15 \%$ del personal científico del país, le correspondió en 1992 sólo el 1,8\% del presupuesto nacional para esta finalidad. En cuanto a fondos concursables, tales como las convocatorias que periódicamente realiza el CONICET, los grupos exclusivamente universitarios compiten con desventaja frente a los que revisten el carácter de institutos incorporados al sistema de este organismo.

A partir de 1976, la participación de las Universidades nacionales en la finalidad de 
ciencia y tecnología del Presupuesto Nacional se redujo casi en un $75 \%$, pasando los fondos sustraídos, a sumarse al presupuesto del CONICET. El resultado es que, por una parte, los laboratorios universitarios se encuentran en una situación claramente desfavorable, de gran debilidad estructural, debido a la baja tasa de inversión en infraestructura científica. Por otra parte, esta medida -que se consolidó en el tiempo- redujo al mínimo la capacidad de las Universidades para orientar su propia investigación.

Debido a ello, a partir de su normalización, las Universidades nacionales vienen desarrollando una activa política de promoción de la investigación científica y tecnológica. Todas ellas han creado Secretarías especializadas en el tema y desde 1985 sus responsables se reúnen en forma sistemática en un cuerpo que originariamente se dio el nombre de CIPCYT y que luego se transformó en asesor de la Comisión de Investigación del CIN.

El progreso de las Universidades en la gestión de las actividades científicas y tecnológicas ha sido muy importante, como así también el grado de coordinación que van alcanzando en la materia. Actualmente se encuentran abocadas al desarrollo de una metodología que permita comparar necesidades y recursos, en orden a una asignación más adecuada del presupuesto para investigación. También en este año han abordado en forma conjunta la evaluación y priorización de proyectos presentados a la cooperación internacional y prevén profundizar esta tarea en 1993 con la organización de cursos de entrenamiento para la formulación de proyectos.

El desarrollo científico y tecnológico de las Universidades debe ser apoyado y estimulado mediante políticas que tiendan a la vinculación de todos los elementos del hoy denominado "sistema nacional de innovación", garantizando -y exigiendo-que ellas ocupen un lugar relevante, ya que:

"el desarrollo de las nuevas tecnologías y el de las industrias modernas de basé científica,
conduce a una nueva valorización de las Universidades como el hugar más adecuado para la
realización de investigaciones científicas y tecnológicas de punta. Este redescubrimiento y
revalorización de la investigación universitaria se debe, entre otros factores, a la superioridad
que las instituciones imiversitarias demuestran frecuentermente, en relación a las empresas o
a institutos aislados, para atraer los mejores talentos y abrir espacios para el ejercicio de la
iniciativa y el liderazgo intelectual en el campo de la ciencia y la tecnología. Otro factor es
el papel de las Universidades como generadoras de nuevas vocaciones y nuevos talentos en
el área cientifico-tecnológica".(SCHWARTZMAN, 1988)

En efecio, en Argentina es probable que el aspecto más relevante de la contribución de las Universidades nacionales al desarrollo científico y tecnológico del país sea el esfuerzo que vienen realizando en materia de formación de investigadores. Muchas Universidades han creado programas de becas de investigación, en los que invierten una parte significativa de los recursos disponibles. El Programa de Becas de Investigación de la UBA es, probablemente, el más importante que ninguna otra institución esté llevando a cabo en el país en la actualidad (cuadro 4).

Desde su creación en 1986, un total de 1565 jóvenes investigadores han sido formados 
por el sistema de becas. Cada uno de ellos ha tenido una permanencia promedio en el sistema, de 24,6 meses. Un programa complementario, de incorporación a la docencia, ha permitido a la UBA que 101 ex becarios ingresen a la Universidad con dedicación exclusiva, lo que contribuye, aunque parcialmente, a revertir el panorama descrito.

\section{La relación de las Universidades con el sector productivo}

En los paises industrializados, la vinculación Universidad-empresa (U-E) pone de manifiesto que una de las características más destacables del actual proceso productivo es el valor estratégico del conocimiento científico y tecnológico. En un proceso de tales características, los laboratorios de investigación básica pueden recibir, con toda propiedad, la denominación de fábricas de conocimiento, acuñada por Jorge Sábato.

En Argentina, la evolución de la relación U-E tiende a ser establecida en un contexto en el que las pautas culturales, el cuadro normativo, la orientación de las políticas y la disponibilidad de instrumentos de promoción, resultan poco favorables a la innovación y al cambio tecnológico.

Muchas dificultades se interponen para el desarrollo de una cultura innovadora, la mayor parte de ellas derivadas de la condición periférica de la economía argentina, las políticas proteccionistas aplicadas durante muchos años, el ajuste recesivo, la dependencia tecnológica de las empresas, la baja tasa de inversión, la escasez de crédito y el peso estructural de la deuda externa, recientemente renegociada. Este contexto explica el hecho de que la relación U-E en nuestro país haya sido impulsada, hasta ahora, con mayor energía desde las propias Universidades, que desde las empresas como supuestas demandantes.

En Argentina, al igual que en otros países, las Universidades que cuentan con capacidad de investigación y de posgrado de alto nivel son más propensas a la relación U-Eque aquellas que se dedican exclusivamente a la docencia, debido a que la capacidad de transferencia está directamente relacionada con la de producir conocimiento científico y tecnológico.

Desde cierta visión ideológica que por momentos parece dominar la escena política argentina se tiende a presentar a las Universidades privadas -que en este país escasamente realizan alguna investigación- como las más adecuadas para colaborar con el sector productivo, confundiendo la naturaleza empresaria de estas Universidades, con lacapacidad de brindar servicios a las empresas. La realidad muestra lo contrario. Son aquellas Universidades públicas que cuentan con mayores recursos científicos las más vinculadas en emprendimientos concretos con el sector productivo.

\section{La cooperación interuniversitaria}

Las Universidades argentinas registran una muy incipiente cooperación interuniversitaria que se desarrolla, o bien en el plano de las relaciones personales de docentes e investigadores, o bien en el de lo político-institucional. Esto deberá ser consolidado en el futuro, ya que la única viabilidad para ciertas Universidades con escasamasacrítica de docentes, investigadores 
$\mathrm{y}$, aun, de estudiantes proviene de la constitución de redes de unidades académicas afines.

En el plano internacional, la cooperación entre Universidades surge como uno de los instrumentos más cficaces para mejorar el nivel de la formación superior y contribuir así a reducir los riesgos de exclusión y marginalidad del país con respecto a las tendencias predominantes en el actual proceso de mundialización.

Las Universidades nacionales se han mostrado muy activas en el campo de la cooperación internacional durante los últimos años. En efecto, la cooperación interuniversitaria a nivel internacional es una condición necesaria para el logro de los objetivos sociales de competitividad y de equidad, dado que permite extender al plano regional el esfuerzo por generar "masa crítica" y aprovecharcomplementariedades, en tanto que -en el plano de la relación con las mejores Universidades de los países industrializadosfavorece el acceso al conocimiento más avanzado.

Otra dimensión de la cooperación interuniversitaria es que resulta un instrumento potente que contribuye a lograr la integración de los países en los planos económico. político y social y así es utilizada, concretamente, en procesos en curso. Un ejemplo de ello es la experiencia del Grupo de Montevideo que agrupa a varias Universidades del área del MERCOSUR. Esta asociación, pese a haber sido creada recientemente, tiende a consolidar sus actividades en forma acelerada.

Por otra parte, además de reforzar los mecanismos integradores regionales, se reconoce a la cooperación interuniversitaria la capacidad de ayudar a definir el perfil y la identidad cultural de la región en el escenario mundializado.

\section{A modo de conclusiones}

El sistema de educación superior argentino afronta la necesidad de una nueva reforma que lo ponga a tono con las transformaciones económicas y sociales que ha experimentado el país en los últimos años y con los desafíos que afronta de cara al futuro. Fenómenos tales como la globalización y mundialización de la economía, la transnacionalización empresarial. las nuevas condiciones de competitividad de los mercados, entre otros aspectos, inducen cambios en la estructura productiva y social del pais y exigen de las Universidades modificaciones sustantivas.

Es preciso rescatar elementos de las más importantes tradiciones universitarias argentinas; entre ellos, el papel indelegable del Estado como elemento de sustentación de la educación superior, y la concepción de Universidad científica, en un marco de autonomía y de libertad académica.

En tal sentido, si el acceso al conocimiento científico y tecnológico es, en nuestra era, una de las principales ventajas comparativas, resulta indudable que las Universidades cuya misión es crear, atesorar y difundir ese conocimiento deben jugar un papel cada vez más importante, para el cual han de estar preparadas. Para ello, las Universidades deben fortalecer sus actividades de $\mathrm{I}+\mathrm{D}$ y estimular el desarrollo de áreas vacantes estratégicas de conocimiento científico, en el marco de las políticas científica y universitaria. con el 
objetivo de crear nuevos conocimientos.

Los requerimientos de desarrollo tecnológico del país hacen que, más allá del valor "esencial" asignado a la ciencia, las Universidades deban desempeñar un papel destacado en lo que actualmente se denomina como sistema nacional de innovación o "sistema ciencia-tecnología-industria". Como productoras de conocimientocientífico tecnológico, las Universidades deben fortalecer su vinculación con el sector productivo.

La cooperación interuniversitaria a nivel internacional es un instrumento imprescindible para el logro de los objetivos sociales de competitividad y de equidad, dado que-en el planoregional-permite generar "masa crítica" y aprovecharcomplementariedades, en tanto que -en el plano de la relación con las mejores Universidades de los países industrializados- favorece el acceso al conocimiento más avanzado.

Se requiere, en consecuencia, promover la cooperación intra y extra-regional, a fin de fortalecercapacidades locales e incorporar el conocimiento científico de avanzada al acervo de las Universidades y Centros de I+D de la región. Es necesario, por ello, estimular la vinculación entre grupos de investigación y docencia de Universidades y Centros de $I+D$, dentro de cada país y a nivel regional, a fin de optimizar, sobre la base de su complementación, las capacidades existentes.

La creación de redes científicas y académicas que faciliten el conocimiento recíproco de los grupos de diferentes países, en temáticas afines, y la conformación de programas concertados de $\mathbf{I}+\mathbf{D}$ constituyen instrumentos clásicos de administración y gestión de la ciencia y la tecnología, que deben ser implementados aprovechando la experiencia acumulada ya en la región.

Esta misión forma parte esencial de la reforma global que debe ser abordada urgentemente como manera de que la sociedad encuentre en sus Universidades un instrumento indispensable para lograr los objetivos del desarrolloeconómicoen condiciones más equitativas.

\section{BIBLIOGRAFIA}

ALBORNOZ, M., Ciencia y Tecnologia en Argentina. Marco canceptual y Panorama General, Infome preparado para el PNUD, editado como documento de trabajo por el CEA, Buenos Aires, noviembre, 1989.

ALBORNOZ, M., "Incentivos al Investigador en Argentina. La carrera del investigador", en lnterciencia, Caracas, diciembre, 1992.

BRUNNER, J.J., Educación Superior en América Latina: cambios y desafios, FCE, Santiago de Chile, 1990. PORTANTIERO, J.C., Estudiantes y Política en América Latina, Siglo XXI. México, 1978.

SCHWARTZMAN, S., Qualidade e relevancia da pesquisa universitaria: 10 teses para discussao, texto presentado para el $2^{\circ}$ Simposio Anual de la SBPC, Sâo Paulo, 1988. 


\section{Cuadro N²1}

Relevamiento de recursos y actividades en Ciencia y Tecnología (RRACYT) - 1988

\begin{tabular}{|c|c|c|}
\hline Sector & Cantidad & $\%$ \\
\hline Universidades(todas) & 10.829 & 56,7 \\
\hline Sector público nacional & 5.232 & 27,3 \\
\hline Sector público provincial & 875 & 4,6 \\
\hline Privado (bien público) & 521 & 2,7 \\
\hline Privado (productivo) & 479 & 2,5 \\
\hline Otros & 1.175 & 6,2 \\
\hline Total & 19.111 & 100,0 \\
\hline
\end{tabular}

Cuadro № 2

Producción de publicaciones científicas
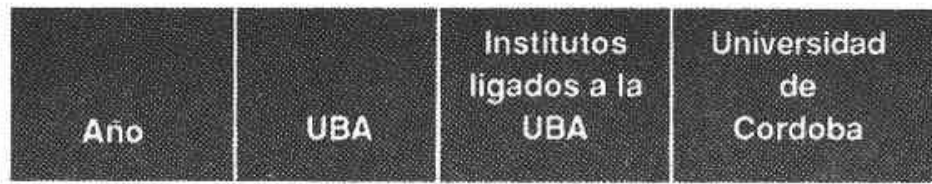

\section{Universidad \\ de \\ La Plata}

1980

354

240

1985

478

137

109

95

1988

536

177

148

157

1990

541

163

161

235

139

268

1991

523

74

153

253

Fuente: Estudio del Lic. Pablo Cedro, para UBACYT. 
Cuadro № 3

Publicaciones de algunas Universidades de América Latina

\begin{tabular}{|c|c|c|c|}
\hline Ano & $\begin{array}{l}\text { Central de } \\
\text { Venzuela }\end{array}$ & San Pablo & $\begin{array}{c}\text { Autonoma de } \\
\text { México }\end{array}$ \\
\hline 1980 & 118 & 588 & 421 \\
\hline 1985 & 90 & 851 & 484 \\
\hline 1989 & 115 & 864 & 535 \\
\hline 1990 & 117 & 1075 & 608 \\
\hline
\end{tabular}

Fuente: Estudio del Lic. Pablo Cedro, para UBACYT.

\section{Cuadro N2 4}

Becarios de Investigación de la UBA

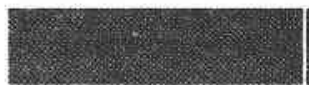

1986

1987

$1988 \quad 1989$

1990

$19911992^{*}$

\begin{tabular}{|c|c|c|c|c|c|c|c|}
\hline estudiantes & 404 & 420 & 430 & 250 & 192 & 191 & 279 \\
\hline iniciación & 155 & 150 & 210 & 150 & 105 & 202 & 288 \\
\hline perfec. . & 64 & 64 & 105 & 71 & 166 & 145 & 204 \\
\hline otal & 623 & 634 & 745 & 471 & 463 & 538 & 771 \\
\hline
\end{tabular}

\footnotetext{
* Hasta el mes de octubre
} 